\title{
A Practical Tessellation-Based Approach for Optimizing Cell-Specific Bias Values in LTE-A Heterogeneous Cellular Networks
}

\author{
Jin-Bae Park and Kwang Soon Kim \\ Department of Electrical and Electronic Engineering, Yonsei University, 50 Yonsei-ro, Seodaemun-gu, \\ Seoul 03722, Republic of Korea
}

Correspondence should be addressed to Kwang Soon Kim; ks.kim@yonsei.ac.kr

Received 26 December 2016; Accepted 22 May 2017; Published 28 June 2017

Academic Editor: Alessandro Tasora

Copyright (C) 2017 Jin-Bae Park and Kwang Soon Kim. This is an open access article distributed under the Creative Commons Attribution License, which permits unrestricted use, distribution, and reproduction in any medium, provided the original work is properly cited.

In order to implement an optimized solution for cell range expansion (CRE) and enhanced intercell interference coordination (eICIC) schemes in long-term evolution-advanced (LTE-A) heterogeneous cellular networks (HCNs) and to realize good loadbalancing performance in existing LTE-A systems, a practical tessellation-based algorithm is proposed. In this algorithm, a globalized cell-specific bias optimization and a localized almost blank subframe (ABS) ratio update are proposed. The proposed scheme does not require major changes to existing protocols. Thus, it can be implemented in existing LTE-A systems with any legacy user equipment (UE) with only a partial update to the BSs and core networks. From simulation results, it is shown that the tessellation formed by the proposed approach is quite consistent with the optimal one for various realistic scenarios. Thus, the proposed scheme can provide a much better load-balancing capability compared with the conventional common bias scheme. Owing to the improved load-balancing capability, the user rate distribution of the proposed scheme is much better than that obtained from the conventional scheme and is even indistinguishable from that of the ideal joint user association scheme.

\section{Introduction}

In current cellular networks, low-powered small cells are being installed with conventional macrocells to form heterogeneous networks (HCNs) as a way to meet users increasing demand for mobile broadband traffic [1]. Considering a cochannel deployment in which macrocells and small cells use the same carrier frequencies and conventional cell association, this may mislead most users by selecting macrocells as the best-serving cells owing to the large difference in downlink transmit powers. Thus, to achieve the potential of such an $\mathrm{HCN}$, appropriate load balancing is essential and is expected to play an important role in offloading loads from macrocells to small cells [2-4]. For this, cell range expansion (CRE) and enhanced intercell interference coordination (eICIC) using almost blank subframes (ABS) have already been adopted in 3GPP long-term evolution-advanced (LTE-A). 3GPP LTE-A allows for offloading even to legacy
UEs and protects such offloaded small-cell users by providing a certain muted portion of subframes so that more users can be offloaded from macrocells to small cells with a significant performance gain [5-7].

There have been many load-balancing schemes in literature to determine the bias values for CRE and the ABS ratio for the eICIC, which can be categorized as follows: (i) a joint optimization of user association and ABS ratios such as in $[8,9]$, (ii) a global optimization of CRE bias values and ABS ratios such as in $[10,11]$, and (iii) a local optimization of ABS ratios for a given common bias value such as in [12-14]. As an ideal approach for CRE and eICIC optimization, joint optimization methods for user association and ABS ratios have been studied $[8,9]$. In [8], a centralized optimization problem for user association considering the ABS ratio is formulated and solved to maximize a network-wide utility and shows a large gain in network performance. In [9], a joint problem for user association, the ABS ratio, and radio 
resource scheduling is studied, and a distributed algorithm is developed. The algorithm shows improvement in networkwide resource utilization. However, in order to implement such a joint approach in an existing network architecture, existing network elements should be updated and legacy UEs need to be modified or even replaced to support this new user association process. In the current LTE-A, user association can adopt the CRE method by using a bias value [15], where a positive bias is assigned to each cell, and each user is associated with the cell of the maximum biased reference signal received power (RSRP). In order to determine a proper bias value and ABS ratio, a global optimization method using cell-specific bias values [10] or a common bias value [11] for small-cell CRE has been studied. In this method, a centralized approach is considered where the CRE bias values and $\mathrm{ABS}$ ratios are determined at a central entity [e.g., the enhanced packet core- (EPC-) mobility management entity (MME)] to provide good performance. Although they may be compatible with legacy UEs, a large amount of overhead is caused by frequent signal exchanges for updating each user's SNR or each BS information. These events are necessary to estimate user rates, and an overall update to the BSs and the core network is also required. As an efficient implementation in an existing network, a local optimization of ABS ratios based on a given common CRE bias value has been also studied [12-14]. In this optimization, simple centralized or distributed algorithms for determining eICIC ABS ratios based on a given common CRE bias value are proposed by comparing local load situations in each macrocell and neighboring small cells. Although this approach can be applied to legacy UEs and requires only a partial update to the BSs and the core network, its performance is limited mainly owing to the use of a common CRE bias value, even without any optimization. Thus, in order to achieve more successful load balancing in existing LTE-A networks, a more efficient method for determining cell-specific bias values and ABS ratios needs to be developed. This method should be implemented without major changes to existing cellular networks.

In this paper, a practical tessellation-based approach is proposed to maximize the network-wide proportional fairness (PF) among users in LTE-A HCNs. In these HCNs, cell-specific bias values are determined to form a suboptimal tessellation similar to an optimal tessellation that may be obtained from a joint optimization for the CRE bias values and $\mathrm{ABS}$ ratios. The proposed scheme does not require any major modifications to existing protocols and thus can be easily applicable to practical LTE-A systems. The proposed algorithm consists of the following two steps: (i) based on the long-term accumulated user measurement information, cell-specific bias values are optimized and updated globally by the EPC-MME in a long-term manner and are delivered infrequently to small cells; and (ii) the ABS ratio and ABS/NS resource scheduling are optimized locally among each $\mathrm{mBS}$ and its associated sBSs only. It is shown that the proposed scheme can be easily realized in practical LTEA systems without modifying any existing protocols, while achieving a good load-balancing performance compared with conventional methods. The rest of this paper is organized as follows. In Section 2, an abstract system model for an LTE-A HCN is provided, and the proposed tessellation-based approach for efficient load balancing in an existing LTE-A $\mathrm{HCN}$ is described in Section 3. In Section 4, simulation results for various realistic scenarios and corresponding discussions are provided, and concluding remarks are given in Section 5.

\section{The System Model}

Consider a downlink two-tier HCN in which macrocells are overlaid with small cells using a lower transmit power. The transmit powers of an $\mathrm{mBS}$ and an sBS are denoted as $P_{m}$ and $P_{s}$, respectively. In addition, the set of user locations, the set of mBS locations, and the set of sBS locations are denoted as $\mathbf{U}=\left\{\mathbf{u}_{1}, \mathbf{u}_{2}, \ldots\right\}, \mathbf{B}^{m}=\left\{\mathbf{b}_{1}^{m}, \mathbf{b}_{2}^{m}, \ldots\right\}$, and $\mathbf{B}^{s}=\left\{\mathbf{b}_{1}^{s}, \mathbf{b}_{2}^{s}, \ldots\right\}$, respectively. Here, each sBS location can be configured for different small-cell service scenarios. For example, in rural environments, sBSs can be deployed in rural communities and remote industries [16]. For urban (dense urban) environments, sBSs can be deployed to support city hot zones or transportation hubs (e.g., airports or railway stations) [17].

The LTE-A core network architecture is abstracted as shown in Figure 1. The sBSs are connected via X2 to the nearest $\mathrm{mBS}$. Here, the nearest $\mathrm{mBS}$ for $\mathrm{sBS} \mathbf{b} \in \mathbf{B}^{s}$ is denoted as $\omega(\mathbf{b})$. In addition, each $\mathrm{mBS}$ or sBS is connected via $\mathrm{S} 1$ to an EPC composed of the MME, the serving gateway (S-GW), and the packet data network gateway (P-GW). In addition, the LTE-A air interface is abstracted so that each subframe of 1 ms intervals consists of $N_{\mathrm{RB}}$ multiple resource blocks (RBs) that are divided into ABSs and normal subframes (NSs). For an ABS, interference from the nearest mBS is almost muted so that the signal quality of small-cell users in the rangeexpanded regions can be greatly improved.

Globally, the EPC-MME determines a set of bias values for sBSs, denoted as $\bar{\kappa}=\left\{\kappa_{\mathbf{b}} \mid \mathbf{b} \in \mathbf{B}^{s}\right\}$. These values are based on long-term accumulated SNR measurement reports from users, where $\kappa_{\mathbf{b}}$ denotes the bias value for $\mathrm{sBS} \mathbf{b}$, which is updated infrequently and delivered to each mBS or sBS. Each user obtains bias information for neighbor sBSs via a system information (SI) block mapped on the radio resource control (RRC) SI message over the downlink shared channel (DL$\mathrm{SCH})$. After receiving the bias values of its neighbor sBSs, each user chooses its serving BS based on the biased RSRP information as

$$
\mathbf{b}_{\mathbf{u}}^{*}=\arg \max _{\mathbf{b} \in \mathbf{B}_{\mathbf{u}}} 10 \log _{10} \gamma_{\mathbf{u}, \mathbf{b}}+\kappa_{\mathbf{b}}
$$

where $\gamma_{\mathbf{u}, \mathbf{b}}$ denotes the averaged received signal-to-noise power ratio (SNR) between $\mathrm{BS} \mathbf{b}$ and user $\mathbf{u}$ and reports its measurement $\gamma_{\mathbf{u}}=\left\{\gamma_{\mathbf{u}, \mathbf{b}} \mid \mathbf{b} \in \mathbf{B}_{\mathbf{u}}\right\}$ and $\mathbf{B}_{\mathbf{u}} \subset \mathbf{B}^{m} \cup \mathbf{B}^{s}$ denotes a neighbor BS list for user $\mathbf{u}$ to the selected BS. Note that (1) becomes conventional cell selection with a maximum RSRP when $\kappa_{\mathbf{b}}=0$ for $\mathbf{b} \in \mathbf{B}^{m} \cup \mathbf{B}^{s}$. Here, denote the set of associated users as $\mathbf{b} \in \mathbf{B}^{m} \cup \mathbf{B}^{s}$ as $\mathbf{U}_{\mathbf{b}}=\left\{\mathbf{u} \in \mathbf{U} \mid \mathbf{b}_{\mathbf{u}}^{*}=\mathbf{b}\right\}$. Based on the current user association results, the ABS ratio $\varsigma_{\mathbf{d}}$ of each $\operatorname{mBS} \mathbf{d} \in \mathbf{B}^{m}$ is optimized locally among each $\mathrm{mBS} \mathbf{d}$ and its local sBSs in $\mathbf{B}_{\mathbf{d}}^{s}=\left\{\mathbf{b}^{\prime} \in \mathbf{B}^{s} \mid \omega\left(\mathbf{b}^{\prime}\right)=\mathbf{d}\right\}$. For each mBS $\mathbf{d} \in \mathbf{B}^{m}$, to determine the ABS ratio $\varsigma_{\mathbf{d}}$, each $\mathrm{mBS} \mathbf{d}$ collects 


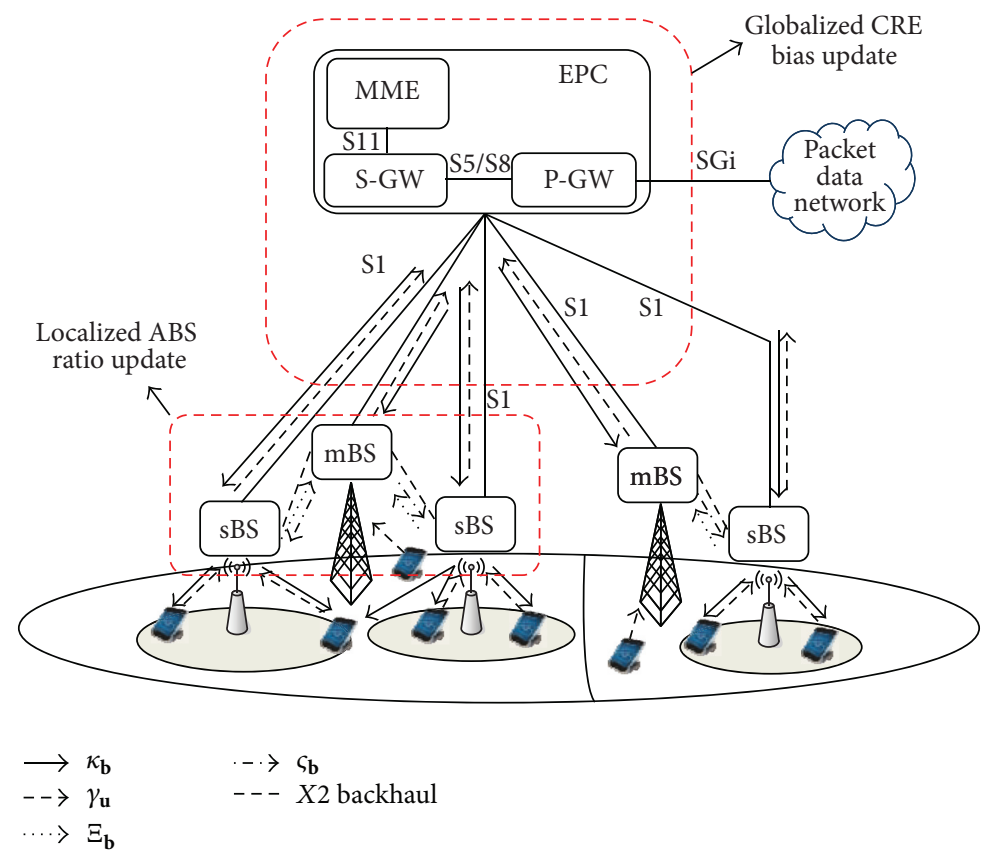

FIgURE 1: Abstract LTE-A HCN model.

all SNR measurement information via the sBS's message $\Xi_{\mathbf{b}}$ from its local sBSs in $\mathbf{B}_{\mathbf{d}}^{s}=\left\{\mathbf{b}^{\prime} \in \mathbf{B}^{s} \mid \omega\left(\mathbf{b}^{\prime}\right)=\mathbf{d}\right\}$. Then, the mBS optimizes the ABS ratio as well as the ABS/NS resource scheduling for each sBS, which is broadcast to each sBS. For user scheduling, each $\mathrm{mBS} \mathbf{b} \in \mathbf{B}^{m}$ can select any users among the associated user set $\mathbf{U}_{\mathbf{b}}$ only in the NS period, while each $\mathrm{sBS} \mathbf{b} \in \mathbf{B}^{s}$ needs to select users among the associated user set $\mathbf{U}_{\mathbf{b}}^{N S}$ (or $\mathbf{U}_{\mathbf{b}}^{\mathrm{ABS}}$ ) only in the NS period (or the ABS period).

\section{Proposed Tessellation-Based Approach for CRE and eICIC Scheme}

In this section, a practical tessellation-based approach is proposed. For this approach, the optimal tessellation of a given system deployment is estimated over a long period, and the cell-specific bias values are determined infrequently to form cell coverage that is as similar to the optimal tessellation as possible in an LTE-A HCN. The proposed approach utilizes existing protocols for data collection and bias value delivery so that it can be easily adopted in a practical LTE-A system. The proposed algorithm consists of the following two steps: (i) based on long-term accumulated user measurement information, cell-specific bias values are updated by using the proposed tessellation-based approach in the EPC-MME over a long period and are delivered infrequently to small cells, and (ii) the ABS ratio and ABS/NS resource scheduling are optimized locally among each mBS and its associated sBSs only.

3.1. Optimal Tessellation. Each BS can deliver its aperiodic SNR measurement reports from its associated users to the EPC-MME via the S1AP management messages [18] so that the EPC-MME can observe and store the reported averaged SNR information for many realizations of user locations. The sample SNR realization at time instance $i$, denoted as $\Gamma(i)=$ $\left\{\left\{\mathbf{u}, \gamma_{\mathbf{u}}\right\} \mid \mathbf{u} \in \mathbf{U}\right\}$, is collected within each given time period. Note that the location information is already available in most smartphone devices, and it is assumed that each BS can attach the location information of associated users to its SNR measurement report.

For a given deployment $\mathbf{B}=\mathbf{B}^{m} \cup \mathbf{B}^{s}$, each cell coverage can be regarded as a tessellation for a given system coverage $\mathbf{R}$, which can be written as $\mathbf{T}=\left\{\mathbf{T}_{\mathbf{b}} \mid \mathbf{b} \in \mathbf{B}\right\} \in \rho(\mathbf{R})$, where $\mathbf{T}_{\mathbf{b}}$ denotes the cell coverage for BS $\mathbf{b} \in \mathbf{B}$ and $\rho(\mathbf{R})$ denotes the collection of all partitions of $\mathbf{R}$. Here, user $\mathbf{u}$ is assumed to be associated with BS $\mathbf{b}$ if $\mathbf{u} \in \mathbf{T}_{\mathbf{b}}$. Then, for a given deployment $\mathbf{B}$ and system coverage $\mathbf{R}$, the optimal tessellation may be formulated as

$$
\mathbf{T}^{*}(\mathbf{B})=\underset{\mathbf{T} \in \rho(\mathbf{R})}{\arg \max } E\left[\sum_{\mathbf{u} \in \mathbf{U}(n)} \log \bar{R}_{\mathbf{u}}(\mathbf{T}) \mid \mathbf{B}\right],
$$

where $\bar{R}_{\mathbf{u}}(\mathbf{T})$ denotes the expected user rate of user $\mathbf{u}$ for a given tessellation $\mathbf{T}$. The expectation is taken over all possible user realizations and channel realizations. However, this expectation is difficult to compute for every candidate tessellation in $\rho(\mathbf{R})$. Thus, instead of solving direct optimization using (2), $\mathbf{T}^{*}(\mathbf{B})$ is estimated from the collected samples of the joint user association results, as summarized in Algorithm 1. In this algorithm, jointly optimized user association results for every SNR realization available at the EPC-MME are obtained and stored. For SNR realization $\Gamma(n)$ at time instance $n$, the expected user rate in the ABS and NS 
(1) Step 0: Initialize $\mathbf{N}$.

(2) Step 1: For each $n \in \mathbf{N}$, solve the following joint UA problem by using $\Gamma(n)$ : $\left\{\mathbf{X}(n),\left\{\varsigma_{\mathbf{b}}(n) \mid \mathbf{b} \in \mathbf{B}^{m}\right\}\right\}$

(3) Step 2: For each $\mathbf{b} \in \mathbf{B}$, set

$$
\begin{aligned}
& =\underset{\left\{\mathbf{X},\left\{\varsigma_{\mathbf{b}} \mid \mathbf{b} \in \mathbf{B}^{m}\right\}\right\}}{\arg \max } \sum_{\mathbf{u} \in \mathbf{U}(n)}\left(\sum_{\mathbf{b} \in \mathbf{B}^{s}} \sum_{\tau \in\{\mathrm{ABS}, \mathrm{NS}\}} x_{\mathbf{u}, \mathbf{b}}^{\tau} \log \frac{\eta_{\mathbf{b}}^{\tau} I_{\mathbf{u}, \mathbf{b}}^{\tau}(n)}{\sum_{\mathbf{u}^{\prime} \in \mathbf{U}(n)} x_{\mathbf{u}^{\prime}, \mathbf{b}}^{\tau}}+\sum_{\mathbf{b} \in \mathbf{B}^{m}} x_{\mathbf{u}, \mathbf{b}} \log \frac{\left(1-\varsigma_{\mathbf{b}}\right) I_{\mathbf{u}, \mathbf{b}}^{\mathrm{NS}}(n)}{\sum_{\mathbf{u}^{\prime} \in \mathbf{U}(n)} x_{\mathbf{u}^{\prime}, \mathbf{b}}}\right), \\
& \text { s.t. } \sum_{\mathbf{u} \in \mathbf{U}(n)}\left(\sum_{\mathbf{b} \in \mathbf{B}^{s}} \sum_{\tau \in\{\mathrm{ABS}, \mathrm{NS}\}} x_{\mathbf{u}, \mathbf{b}}^{\tau}+\sum_{\mathbf{b} \in \mathbf{B}^{m}} x_{\mathbf{u}, \mathbf{b}}\right)=1 \text {, } \\
& x_{\mathbf{u}, \mathbf{b}} \in[0,1], \mathbf{u} \in \mathbf{U}(n), \mathbf{b} \in \mathbf{B}^{m}, x_{\mathbf{u}, \mathbf{b}}^{\mathrm{ABS}}, x_{\mathbf{u}, \mathbf{b}}^{\mathrm{NS}} \in[0,1], \mathbf{u} \in \mathbf{U}(n), \mathbf{b} \in \mathbf{B}^{s}, \\
& \varsigma_{\mathbf{b}} \in[0,1], \mathbf{b} \in \mathbf{B}^{m}, \\
& \text { where } \mathbf{X}(n)=\left\{x_{\mathbf{u}, \mathbf{b}}^{\tau}(n) \mid \tau \in\{\mathrm{ABS}, \mathrm{NS}\}, \mathbf{u} \in \mathbf{U}(n), \mathbf{b} \in \mathbf{B}^{s}\right\} \\
& \cup\left\{x_{\mathbf{u}, \mathbf{b}}(n) \mid \mathbf{u} \in \mathbf{U}(n), \mathbf{b} \in \mathbf{B}^{m}\right\} .
\end{aligned}
$$

$$
\begin{aligned}
& \quad \mathbf{U}_{\mathbf{b}}(n)=\left\{\mathbf{u} \in \mathbf{U}(n) \mid x_{\mathbf{u}, \mathbf{b}}(n) \geq x_{\mathbf{u}, \mathbf{b}^{\prime}}(n) \text { for } \forall \mathbf{b}^{\prime} \in \mathbf{B}\right\}, \\
& \text { where } x_{\mathbf{u}, \mathbf{b}}(n)=\max _{\tau \in\{\mathrm{ABS}, \mathrm{NS}\}} x_{\mathbf{u}, \mathbf{b}}^{\tau}(n) .
\end{aligned}
$$

(4) Step 3: Estimate the optimal tessellation as

$$
\begin{aligned}
& \quad \mathbf{T}^{*}(\mathbf{B})=\left\{\mathbf{T}_{\mathbf{b}}=\left\{\mathbf{t} \in \mathbf{R} \mid w(\mathbf{t}, \mathbf{b}) \geq w\left(\mathbf{t}, \mathbf{b}^{\prime}\right) \text { for } \forall \mathbf{b}^{\prime} \in \mathbf{B}\right\} \mid \mathbf{b} \in \mathbf{B}\right\}, \\
& \text { where } w(\mathbf{t}, \mathbf{b})=\sum_{\mathbf{u} \in \mathbf{U}_{\mathbf{b}}(n), n \in \mathbf{N}} f(\|\mathbf{t}-\mathbf{u}\|) \text { for a kernel function } f(x)=(1 / \sqrt{2 \pi} \sigma) e^{-x^{2} / 2 \sigma^{2}} .
\end{aligned}
$$

Algorithm 1: Proposed estimation method for optimal tessellation.

of user $\mathbf{u}$ if associated with BS $\mathbf{b}, I_{\mathbf{u}, \mathbf{b}}^{\tau}(n)$ for $\tau=\{\mathrm{ABS}, \mathrm{NS}\}$, is approximated as

$$
\begin{aligned}
I_{\mathbf{u}, \mathbf{b}}^{\tau}(n)= & \log _{2}\left(1+\frac{E_{S}(\mathbf{b})}{E_{I}^{\tau}(\mathbf{b})}\right) \\
& +\left(\frac{E_{S}^{2}(\mathbf{b})+V_{I}^{\tau}(\mathbf{b})}{\left(E_{S}(\mathbf{b})+E_{I}^{\tau}(\mathbf{b})\right)^{2}}-\frac{V_{I}^{\tau}(\mathbf{b})}{\left(E_{I}^{\tau}(\mathbf{b})\right)^{2}}\right) \log _{2} e
\end{aligned}
$$

where $\eta_{\mathbf{b}}^{\mathrm{ABS}}=\varsigma_{\omega(\mathbf{b})}, \eta_{\mathbf{b}}^{\mathrm{NS}}=1-\varsigma_{\omega(\mathbf{b})}$ for $\mathbf{b} \in \mathbf{B}^{s}, E_{S}(\mathbf{b}) \triangleq$ $\gamma_{\mathbf{u}, \mathbf{b}}, E_{I}^{\tau}(\mathbf{b}) \triangleq \sum_{\mathbf{b}^{\prime} \in \mathbf{B}_{\mathbf{u}}-\{\mathbf{b}\}} \gamma_{\mathbf{u}, \mathbf{b}^{\prime}}-\mathbf{1}_{\{\tau=\mathrm{ABS}\}} \gamma_{\mathbf{u}, \omega(\mathbf{b})}$, and $V_{I}^{\tau}(\mathbf{b}) \triangleq$ $\sum_{\mathbf{b}^{\prime} \in \mathbf{B}_{\mathbf{u}}-\{\mathbf{b}\}} \gamma_{\mathbf{u}, \mathbf{b}^{\prime}}^{2}-\mathbf{1}_{\{\tau=\mathrm{ABS}\}} \gamma_{\mathbf{u}, \omega(\mathbf{b})}^{2}$. Here, (3) adopts the Gamma distribution approximation as in [19] with the assumption that the total interference power can be approximated as the sum of the average power from interfering BSs. Note that the optimization problem in Step 1 is not convex but has a special structure that lets the problem become convex in $\mathbf{X}$ for a given $\left\{\varsigma_{\mathbf{b}} \mid \mathbf{b} \in \mathbf{B}^{m}\right\}$ and vice versa. Thus, $\mathbf{X}$ and $\left\{\varsigma_{\mathbf{b}} \mid \mathbf{b} \in \mathbf{B}^{m}\right\}$ can be found by fixing each other and using a convex programming tool such as CVX [20] iteratively. From the relaxed association result $\mathbf{X}(n)$, the set of sample points $\left\{\mathbf{U}_{\mathbf{b}}(n) \mid \mathbf{b} \in \mathbf{B}\right\}$ for estimating the optimal tessellation is obtained. Then, from the obtained sample point sets over a long period $\mathbf{N}$, the optimal tessellation is estimated as in Step 3 by using a kernel function $f(x)$. Here, a Gaussian kernel function with mean of 0 and variance of $\sigma^{2}=4$ is used, but other kernel functions can also be applied.

\subsection{Suboptimal Tessellation Formed by Using Cell-Specific Bias} Values. In this subsection, based on the estimated optimal tessellation from the previous subsection, the cell-specific bias values, denoted as $\bar{\kappa}=\left\{\kappa_{\mathbf{b}} \mid \mathbf{b} \in \mathbf{B}^{s}\right\}$, are determined to form cell coverage that is as similar to the estimated optimal tessellation as possible. Here, $\kappa_{\mathbf{b}}=0$ for $\mathbf{b} \in \mathbf{B}^{m}$ is assumed. Denote the tessellation formed by applying CRE with bias values in $\bar{\kappa}$ for a given deployment $\mathbf{B}$ as $\mathbf{T}(\bar{\kappa}, \mathbf{B})$, which can be written as

$$
\begin{gathered}
\mathbf{T}(\bar{\kappa}, \mathbf{B})=\left\{\mathbf{T}_{\mathbf{b}}(\bar{\kappa}, \mathbf{B})=\left\{\mathbf{t} \in \mathbf{R} \mid 10 \log _{10} \gamma_{\mathbf{t}, \mathbf{b}}+\kappa_{\mathbf{b}}\right.\right. \\
\left.\left.\geq 10 \log _{10} \gamma_{\mathbf{t}, \mathbf{b}^{\prime}}+\kappa_{\mathbf{b}^{\prime}} \text { for } \forall \mathbf{b}^{\prime} \in \mathbf{B}\right\} \mid \mathbf{b} \in \mathbf{B}\right\},
\end{gathered}
$$

where $\gamma_{\mathbf{t}, \mathbf{b}}$ denotes the SNR information of BS $\mathbf{b} \in \mathbf{B}$ measured at $\mathbf{t} \in \mathbf{R}$. Then, the cell-specific bias values may be determined as

$$
\overline{\mathcal{\kappa}}^{*}=\underset{\bar{\kappa}}{\arg \max } \sum_{\mathbf{b} \in \mathbf{B}}\left|\mathbf{T}_{\mathbf{b}}^{*}(\mathbf{B}) \cap \mathbf{T}_{\mathbf{b}}(\overline{\mathcal{\kappa}}, \mathbf{B})\right|
$$

Since the optimal bias value set in (5) is difficult to compute directly, a suboptimal algorithm using the hill-climbing method with a random walk [21] is applied, which is summarized in Algorithm 2. Here, the consistency value $\xi_{\mathbf{b}}\left(\bar{\kappa}^{j}\right)$, defined as the cardinality (area) of the intersection between the cell coverage of BS $\mathbf{b}$ in the optimal tessellation and that formed by applying CRE with bias values in $\bar{\kappa}$, is utilized. In each iteration, the BS with the lowest consistency value is selected in Step 2, and the cell-specific bias value of the selected BS is updated in a greedy manner. In Step 5, a new bias value set is accepted if it is better than the currently best bias value set. A random neighbor for the next iteration is generated in Step 6. After a sufficient number of iterations, the cell-specific bias values for the suboptimal tessellation are determined and then are delivered to the corresponding cells.

3.3. Localized ABS Ratio and Resource Scheduling Optimization. After receiving $\kappa_{\mathbf{b}}$, each sBS $\mathbf{b}$ updates its bias value and broadcasts it via the SI block mapped on the RRC SI message over DL-SCH [22]. Then, each user chooses a serving BS as in (1) by using the biased RSRP information of its neighbor sBSs. Based on the user association results, the ABS ratio $\varsigma_{\mathbf{d}}$ of each $\mathrm{mBS} \mathbf{d} \in \mathbf{B}^{m}$ is optimized by each $\mathrm{mBS} \mathbf{d}$ locally. To 
(1) Step 0: Initialize $\bar{\kappa}^{j}=\left\{\kappa_{\mathbf{b}}^{j}=0 \mid \mathbf{b} \in \mathbf{B}^{s}\right\}$ for $j=1, \ldots,\left|\mathbf{B}^{s}\right|, l=0, l_{\max } \geq 30, \xi_{\text {best }}=0, \Delta_{\text {bias }}(=0.5)$.

(2) Step 1: Set $j=0$ and $\mathbf{L}^{1}=\mathbf{B}^{s}$.

(3) Step 2: Increase $j$ by 1 and select the sBS with the minimum value by calculating the degree of consistency for each $\mathbf{b} \in \mathbf{L}^{j}, \xi_{\mathbf{b}}\left(\overline{\mathcal{\kappa}}^{j}\right)=\left|\mathbf{T}_{\mathbf{b}}^{*}(\mathbf{B}) \cap \mathbf{T}_{\mathbf{b}}^{j}\left(\overline{\mathcal{\kappa}}^{j}, \mathbf{B}\right)\right|$, as

$$
\widehat{\mathbf{b}}=\underset{\mathbf{b} \in \mathbf{L}^{j}}{\arg \min } \xi_{\mathbf{b}}\left(\bar{\kappa}^{j}\right) \text {. }
$$

(4) Step 3: For $\widehat{\mathbf{b}}$, repeat the following process until $\xi\left(\bar{\kappa}^{j}\right)=\sum_{\mathbf{b} \in \mathbf{B}} \xi_{\mathbf{b}}\left(\bar{\kappa}^{j}\right)$ cannot be further maximized. If an increase in $\kappa_{\widehat{\mathbf{b}}}^{j}$ results in a better consistency value such that $\xi\left(\bar{\kappa}_{\widehat{\mathbf{b}},+}^{j}\right)>\xi\left(\bar{\kappa}^{j}\right)$, set $\bar{\kappa}^{j}=\bar{\kappa}_{\widehat{\mathbf{b}},+}^{j}$. Otherwise, if a decrease in $\kappa_{\widehat{\mathbf{b}}}^{j}$ results in a better consistency value such that $\xi\left(\bar{\kappa}_{\widehat{\mathbf{b}},-}^{j}\right)>\xi\left(\bar{\kappa}^{j}\right)$, set $\bar{\kappa}^{j}$ $=\bar{\kappa}_{\widehat{\mathbf{b}},-}^{j}$. Here $\bar{\kappa}_{\widehat{\mathbf{b}},+}^{j}=\left\{\kappa_{\mathbf{b}}^{j}\right\}_{\mathbf{b} \in \mathbf{B}^{s} / \widehat{\mathbf{b}}} \cup\left\{\kappa_{\widehat{\mathbf{b}}}^{j}+\Delta_{\text {bias }}\right\}$ and $\bar{\kappa}_{\widehat{\mathbf{b}},-}^{j}=\left\{\kappa_{\mathbf{b}}^{j}\right\}_{\mathbf{b} \in \mathbf{B}^{s} / \widehat{\mathbf{b}}} \cup\left\{\kappa_{\widehat{\mathbf{b}}}^{j}-\Delta_{\text {bias }}\right\}$.

(5) Step 4: If $\mathbf{L}^{j} \neq \emptyset$, set $\mathbf{L}^{j+1}=\mathbf{L}^{j}-\{\widehat{\mathbf{b}}\}$ and $\bar{\kappa}^{j+1}=\bar{\kappa}^{j}$ and go back to Step 2.

(6) Step 5: If $\xi\left(\overline{\mathcal{\kappa}}^{\left|\mathbf{B}^{s}\right|}\right)>\xi_{\text {best }}$, set $\overline{\mathcal{\kappa}}^{*}=\bar{\kappa}^{\left|\mathbf{B}^{s}\right|}$ and $\xi_{\text {best }}=\xi\left(\overline{\mathcal{\kappa}}^{\left|\mathbf{B}^{s}\right|}\right)$.

(7) Step 6: If $l<l_{\text {max }}$, go back to Step 1 by setting $\bar{\kappa}^{1}=\varepsilon\left(\bar{\kappa}^{*}\right)$ and increasing $l$ by 1 . Otherwise, terminate the algorithm. Here, $\varepsilon(\bar{\kappa})$ generates a random neighbor around $\bar{\kappa}$.

Algorithm 2: Proposed algorithm for determining cell-specific bias values.

do that, each mBS $\mathbf{d} \in \mathbf{B}^{m}$ collects the SNR measurement information $\Xi_{\mathbf{b}}=\left\{\gamma_{\mathbf{u}} \mid \mathbf{u} \in \mathbf{U}_{\mathbf{b}}\right\}$ from its neighboring sBSs $\mathbf{B}_{\mathbf{d}}^{s}$ via X2 interfaces. Then, based on $\left\{\Xi_{\mathbf{b}} \mid \mathbf{b} \in \mathbf{B}_{\mathbf{d}}^{s}\right\}$, the following optimization problem is solved periodically at $\mathrm{mBS} \mathbf{d}$ :

$$
\begin{aligned}
&\left\{\mathbf{X}_{\mathbf{d}}^{*}, \varsigma_{\mathbf{d}}^{*}\right\}=\underset{\left\{\mathbf{X}_{\mathbf{d}}, \varsigma_{\mathbf{d}}\right\}}{\arg \max } \sum_{\mathbf{b} \in \mathbf{B}_{\mathbf{d}}^{s}} \sum_{\mathbf{u} \in \mathbf{U}_{\mathbf{b}}} \sum_{\tau \in\{\mathrm{ABS}, \mathrm{NS}\}} x_{\mathbf{u}, \mathbf{b}}^{\tau} \log \frac{\varsigma_{\mathbf{d}} I_{\mathbf{u}, \mathbf{b}}^{\tau}}{\sum_{\mathbf{u}^{\prime} \in \mathbf{U}_{\mathbf{b}}} x_{\mathbf{u}^{\prime}, \mathbf{b}}^{\tau}}+\sum_{\mathbf{u} \in \mathbf{U}_{\mathbf{d}}} \log \frac{\left(1-\varsigma_{\mathbf{d}}\right) I_{\mathbf{u}, \mathbf{d}}^{\mathrm{NS}},}{\left|\mathbf{U}_{\mathbf{d}}\right|}, \\
& \text { s.t. } x_{\mathbf{u}, \mathbf{b}}^{\mathrm{NS}}+x_{\mathbf{u}, \mathbf{b}}^{\mathrm{ABS}}=1, \\
& x_{\mathbf{u}, \mathbf{b}}^{\mathrm{ABS}}, x_{\mathbf{u}, \mathbf{b}}^{\mathrm{NS}} \in[0,1], \\
& \mathbf{u} \in \mathbf{U}_{\mathbf{b}}, \\
& \mathbf{b} \in \mathbf{B}_{\mathbf{d}}^{s}, \\
& \varsigma_{\mathbf{d}} \in[0,1],
\end{aligned}
$$

where $\mathbf{X}_{\mathbf{d}}=\left\{x_{\mathbf{u}, \mathbf{b}}^{\tau} \mid \tau \in\{\right.$ ABS, NS $\left.\}, \mathbf{u} \in \mathbf{U}_{\mathbf{b}}, \mathbf{b} \in \mathbf{B}_{\mathbf{d}}^{s}\right\}$. Note that the above optimization problem is not convex, but it has a special structure that lets the problem become convex in $\mathbf{X}_{\mathbf{d}}$ for a given $\varsigma_{\mathbf{d}}$, and vice versa, so that $\mathbf{X}_{\mathbf{d}}$ and $\boldsymbol{\zeta}_{\mathbf{d}}$ can be found by fixing each other and using a convex programming tool such as CVX [20] iteratively. From the relaxed association result $\mathbf{X}_{\mathbf{d}}, \mathbf{U}_{\mathbf{b}}^{\mathrm{NS}}$ and $\mathbf{U}_{\mathbf{b}}^{\mathrm{ABS}}$ are obtained as $\mathbf{U}_{\mathbf{b}}^{\mathrm{NS}}=\left\{\mathbf{u} \in \mathbf{U}_{\mathbf{b}} \mid\right.$ $\left.x_{\mathbf{u}, \mathbf{b}}^{\mathrm{NS}} \geq x_{\mathbf{u}, \mathbf{b}}^{\mathrm{ABS}}\right\}$ and $\mathbf{U}_{\mathbf{b}}^{\mathrm{ABS}}=\mathbf{U}_{\mathbf{b}}-\mathbf{U}_{\mathbf{b}}^{\mathrm{NS}}$, respectively. Then, the determined ABS ratio value and user association results are delivered to the corresponding sBS.

For user scheduling, each $\mathrm{mBS} \mathbf{b} \in \mathbf{B}^{m}$ can select any users among the associated user set $\mathbf{U}_{\mathbf{b}}$ only in the NS period, while each sBS $\mathbf{b} \in \mathbf{B}^{s}$ needs to select users among the associated user set $\mathbf{U}_{\mathbf{b}}^{\mathrm{NS}}$ ( or $\mathbf{U}_{\mathbf{b}}^{\mathrm{ABS}}$ ) only in the NS period (or the ABS period).

\section{Simulation Results}

For realistic LTE-A HCN deployment scenarios, the rural [16], urban [17], and dense (or ultradense) urban [23] scenarios are assumed. In the rural scenario, mBSs are deployed regularly to cover a wide area with a long intersite distance (ISD) [e.g., $1732(\mathrm{~m})$ ] [24], and 5-30 sBSs per $\mathrm{mBS}$ are sparsely deployed across the entire macrocell area (excluding locations very close to the macrocells) [25]. For the urban scenario, dense mBSs are deployed irregularly with a minimum distance from other BSs [e.g., at least $2 *$ (cell radius) from sBSs] [26], and a single sBS or a few sBSs are deployed to primarily serve as hotspots such as indoor shopping malls or transportation hubs. For the dense urban scenario, denser small-cell deployment (e.g., 10 or more sBSs [27]) for each given localized hotspot region is considered to support wireless traffic demand more aggressively in such hotspots.

In order to obtain deployment realizations and various SNR realizations per a given deployment for each scenario, appropriate point process models [28] are used to model the locations of mBSs, sBSs, and users on a given $4000-\mathrm{m} \times$ 4000- $\mathrm{m}$ area according to the statistical characteristics of each scenario. For the rural scenario, since the rural mBS 


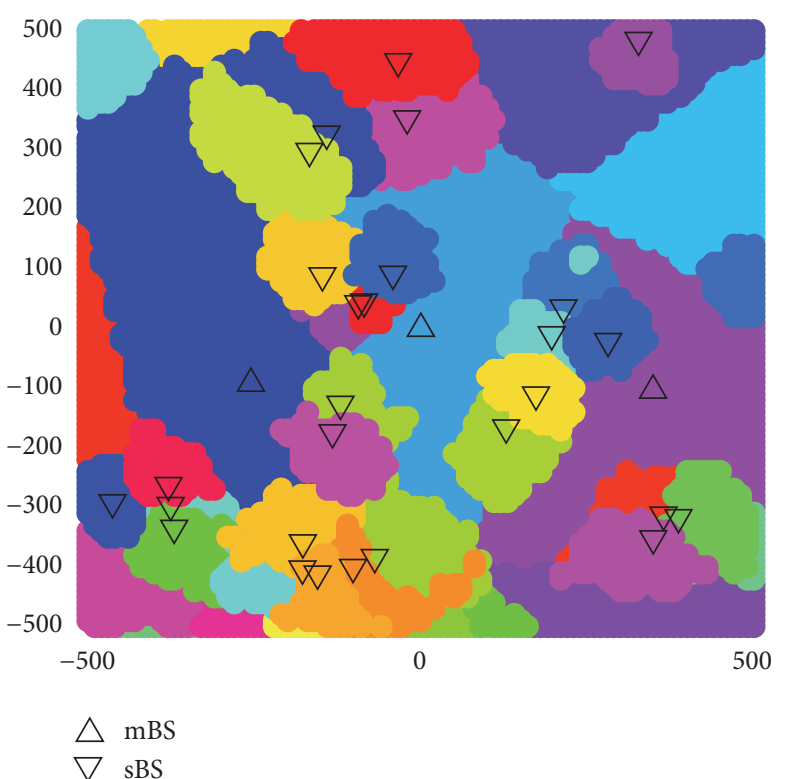

(a) Optimal tessellation

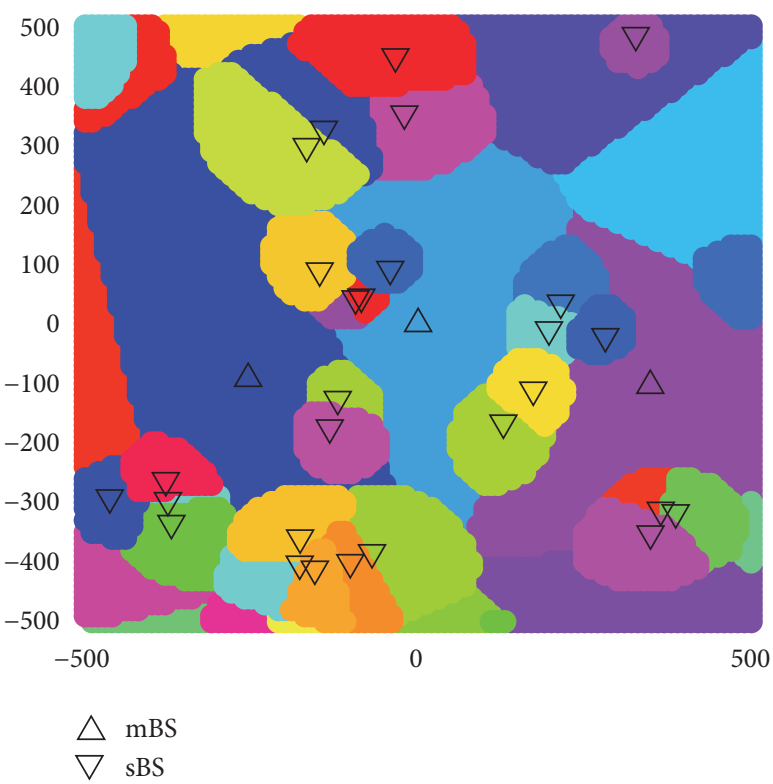

(c) Tessellation using common bias

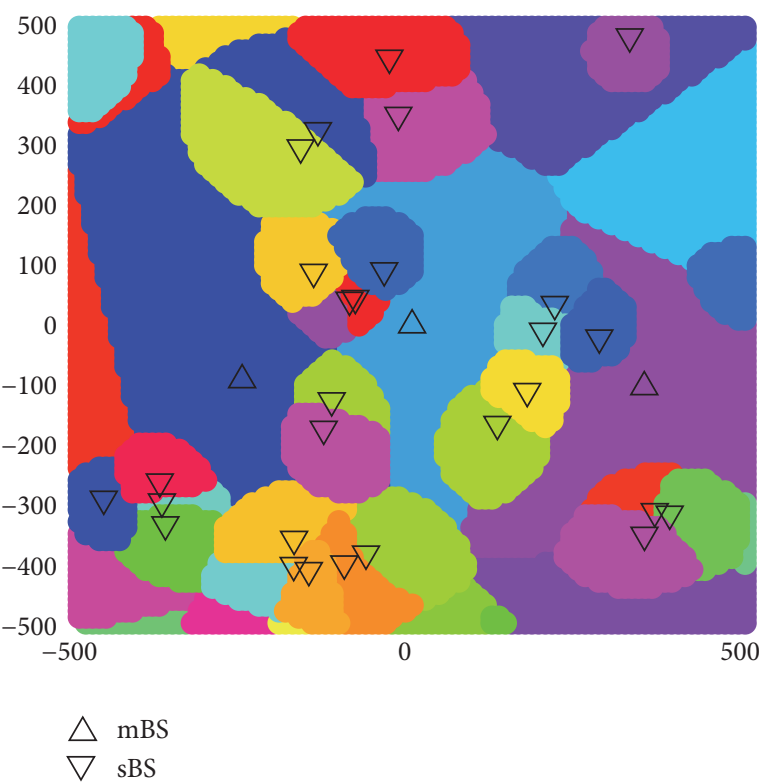

(b) Tessellation using cell-specific bias values

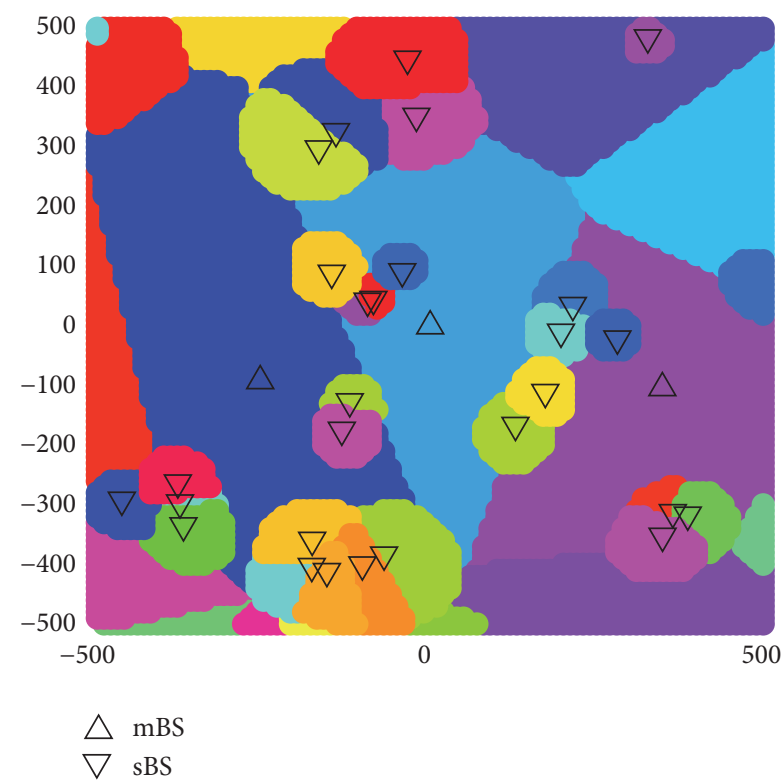

(d) Tessellation using no bias

Figure 2: Tessellation examples for urban scenario.

deployment is quite regular and the sBSs are typically sparse and isolated, $\mathrm{mBS}$ locations are modeled as typical hexagonal cells. Meanwhile, sBS locations are modeled by a Matérn hardcore process of type 3 with a density of $\lambda_{s}$ generated from a homogeneous Poisson point process (HPPP). For the urban or dense urban scenario, since mBSs are deployed irregularly and sBSs are deployed to serve local hotspots, a clustered HCN is considered as in [29], in which a set of cluster center points $\mathbf{C}=\left\{\mathbf{c}_{1}, \mathbf{c}_{2}, \ldots\right\}$ is modeled by a Matérn hardcore process of type 3 with a density of $\lambda_{c}$ generated from an HPPP with the constraint that each cluster center is not located within a predetermined distance from any other cluster center or any mBS. In addition, sBSs for each cluster $\mathbf{c}$, denoted as $\mathbf{B}_{\mathbf{c}}^{s}$, are generated from an HPPP with a density of $\lambda_{s}(\mathbf{c})$ over a circular region of radius $R_{c}$ centered at $\mathbf{c}$, which results in $\mathbf{B}^{s}=\bigcup_{c \in C} \mathbf{B}_{c}^{s}$. The mBS location set $\mathbf{B}^{m}$ is also generated by a Matérn hardcore process of type 3 with a density of $\lambda_{m}$ generated from an HPPP with the constraint that a macrocell BS is not located within a predetermined distance from any cluster center. To evaluate the performance of the proposed approach, for the rural scenario, the ISD for the hexagonal cell model is set to $1732(\mathrm{~m})$, and $5 \mathrm{sBSs}$ per $\mathrm{mBS}$ in average are generated. For the urban and the dense urban scenarios, $\lambda_{m}=4 \times 10^{-6}\left(\mathrm{~m}^{-2}\right), \lambda_{c}=8 \times 10^{-6}\left(\mathrm{~m}^{-2}\right)$, 


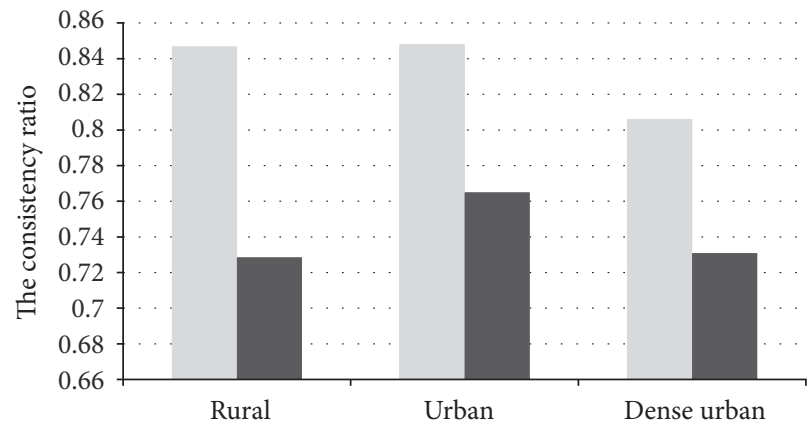

Proposed scheme

- Common bias scheme

FIGURE 3: Average consistency ratio.
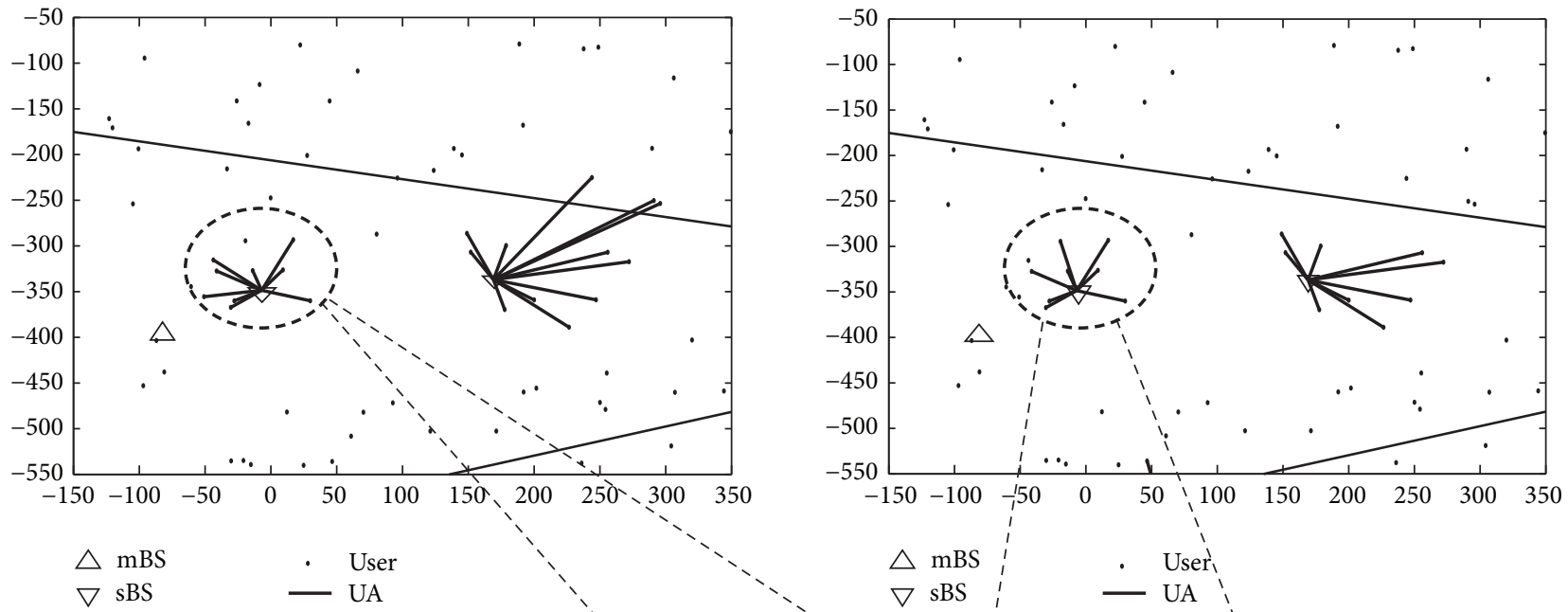

$\nabla$ sBS

(a) Joint UA scheme

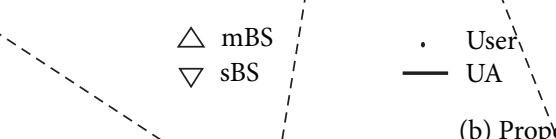

b) Proposed scheme

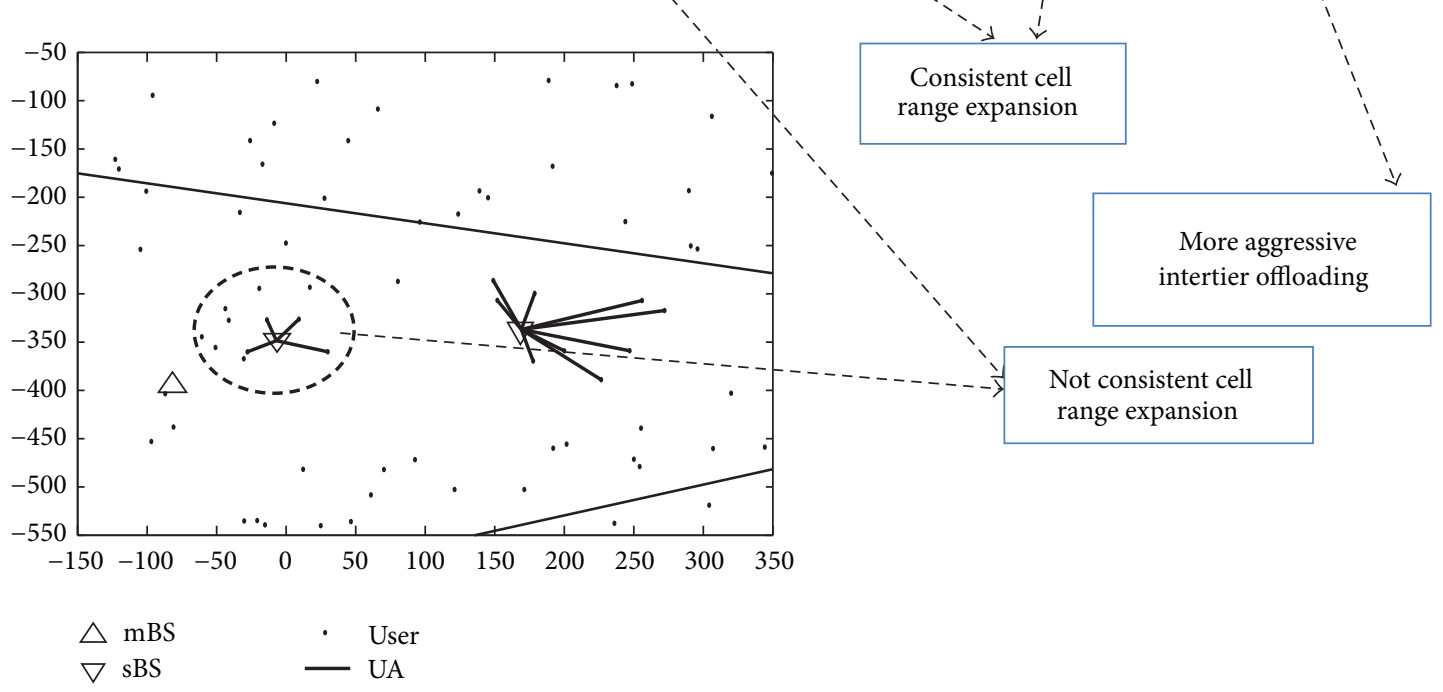

(c) Common bias scheme

FIgURE 4: Typical UA association examples for rural scenario. 


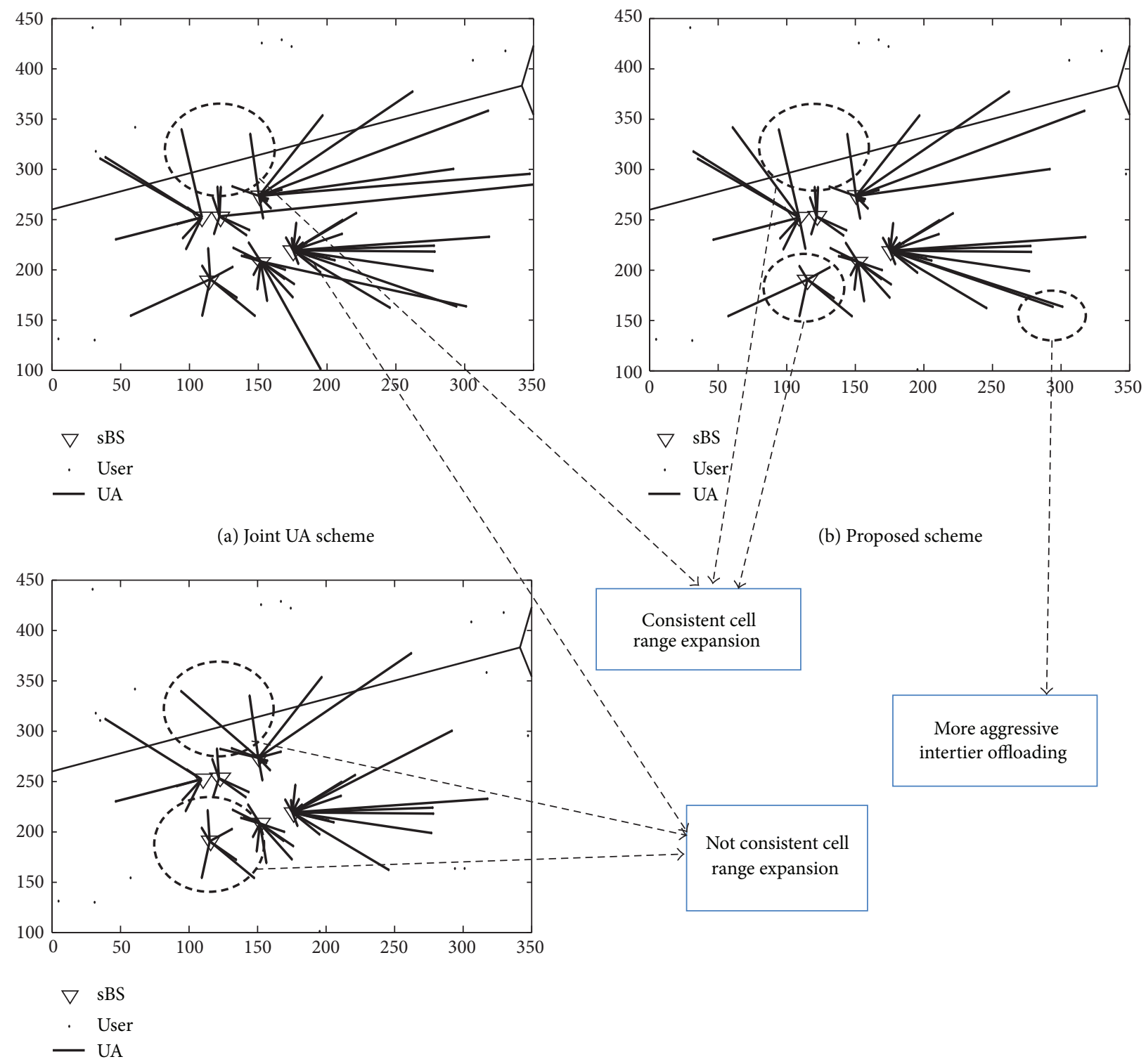

(c) Common bias scheme

FIgURE 5: Typical UA association examples for urban scenario.

$R_{c}=65(\mathrm{~m})$, the minimum distance between a macrocell $\mathrm{BS}$ and a cluster center and that between neighboring cluster centers are set to $1.5 R_{c}$ and $2 R_{c}$, and 3 and $10 \mathrm{sBSs}$ on average for each small-cell cluster are deployed, that is, $\pi R_{c}{ }^{2} \lambda_{s}(\mathrm{c})=$ 3 and $\pi R_{c}{ }^{2} \lambda_{s}(\mathbf{c})=10$, respectively. For user locations, in the rural and urban scenarios, the set of user locations is generated from an HPPP with density of $7.7 \times 10^{-5}$ (users $/ \mathrm{m}^{2}$ ) and $4 \times 10^{-4}$ (users $/ \mathrm{m}^{2}$ ), respectively, while, in the dense urban scenario, the set of user locations is generated from a mixture point process $\Phi^{u} \cup \bigcup_{\mathbf{c} \in \mathbf{C}} \Phi_{\mathrm{c}}^{u}$, where $\Phi^{u}$ is an HPPP with a density of $\lambda_{u}=4 \times 10^{-4}\left(\mathrm{~m}^{-2}\right)$ and $\Phi_{c}^{u}$ is an HPPP over the circular region centered at $\mathrm{c}$ with a density of $\lambda_{h}=10 \lambda_{u}$. In [30], it is shown that a typical hotspot area size follows a Weibull distribution with parameters $\lambda=8.14$ and $k=0.89$ for hotspots having more than five times the mean traffic of a normal area, by which a typical mean value of 10 for the user density ratio and $R_{c}=65(\mathrm{~m})$ corresponding to the upper $20 \%$ of the area sizes are picked for the simulation setup. The transmit powers of the mBSs and sBSs are set to $46 \mathrm{dBm}$ and $23 \mathrm{dBm}$, respectively; the noise power spectral density is set to $-174 \mathrm{dBm} / \mathrm{Hz}$; and the system bandwidth is assumed to be $10 \mathrm{MHz}$. In addition, $N_{\mathrm{RB}}=100$ and the channel for each RB between each BS and each user is assumed to be an independent flat Rayleigh fading channel with a path-loss exponent of $\alpha=4$. For comparison, the centralized joint scheme for UA and ABS ratios [8], denoted as "Joint," is considered to provide an upper bound on the performance, in which all information is assumed to be available at the EPCMME and the joint optimization is performed by considering the user locations jointly. In addition to the "Joint" scheme, 


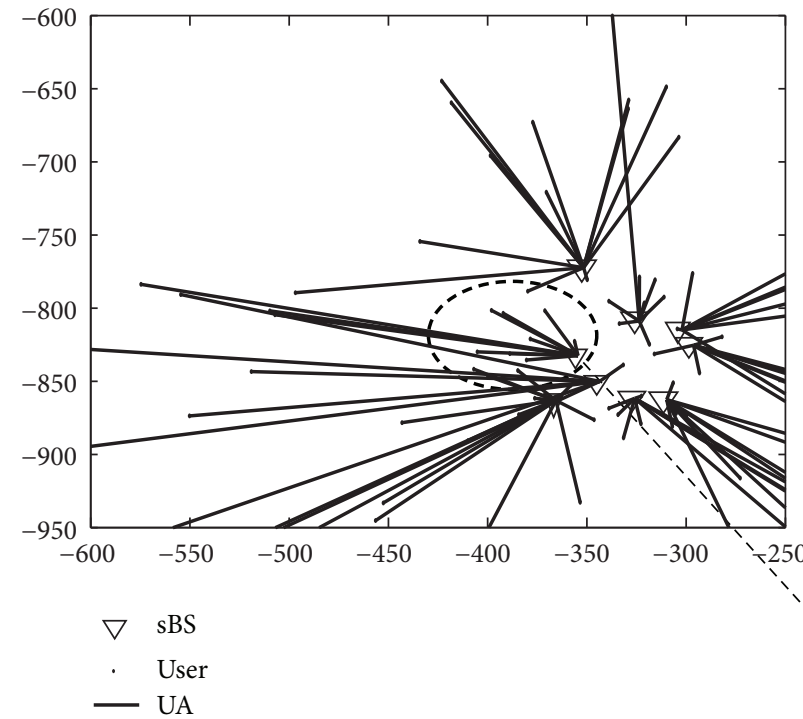

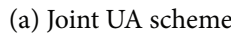

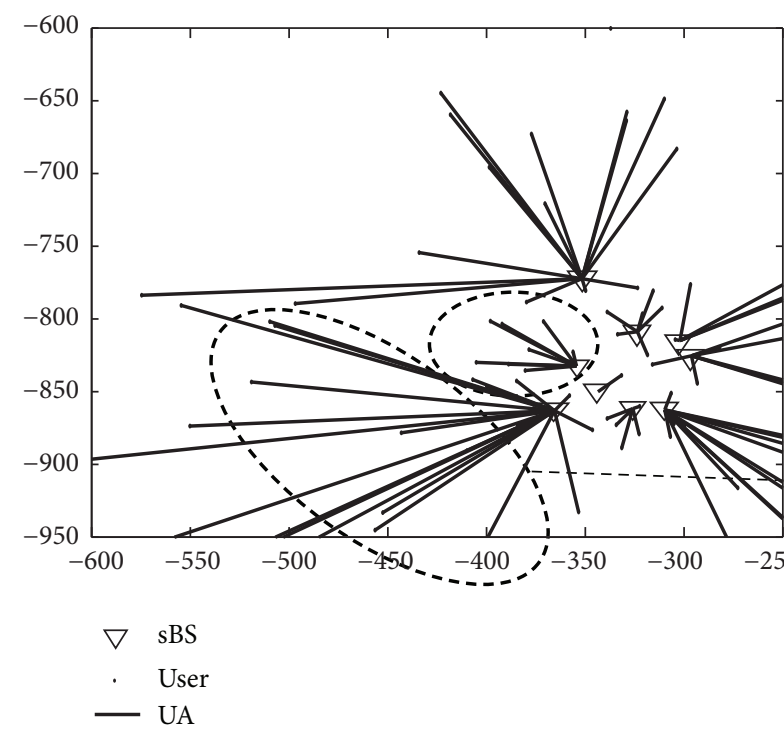

(c) Common bias scheme

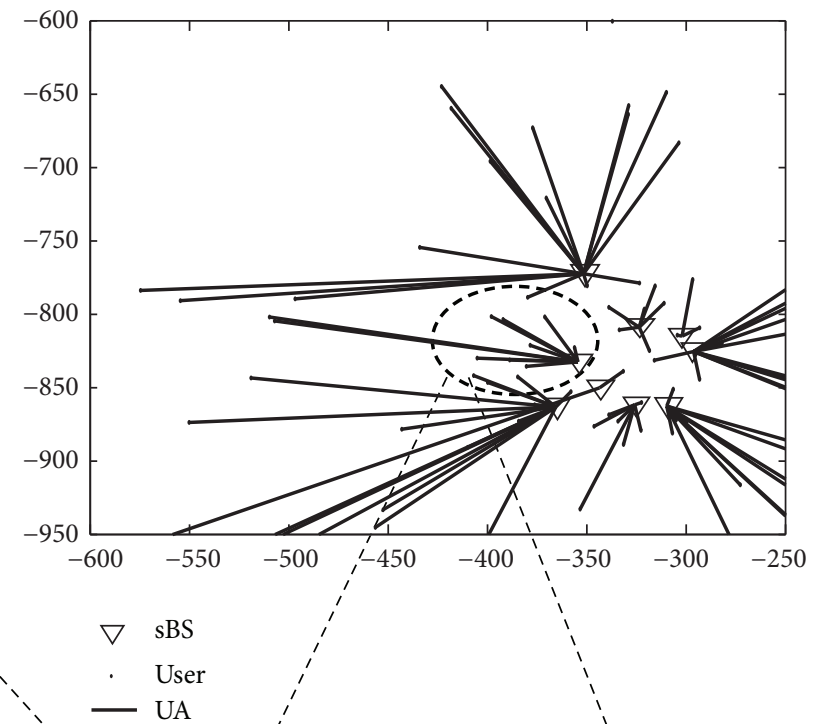

Figure 6: Typical UA association examples for dense urban scenario.

a scheme using a common bias value and the maximum RSRP scheme with no bias are also compared. They are denoted as "Common" and "MAX," respectively. Here, for the common bias scheme, the common bias value is set to a typical value of $6 \mathrm{~dB}$ [31], and the same localized ABS and resource scheduling optimization are applied as in the proposed scheme.

In Figure 2, some typical tessellation examples for the urban scenario are illustrated. These examples exhibit an improved similarity to the optimal tessellation of the proposed scheme over the common and no-bias schemes. To quantify such an improvement achieved by using the proposed scheme, the normalized sum consistency value of sBSs (by the total sBS area in the optimal tessellation) of the proposed scheme is compared with that of the common bias scheme in the three realistic scenarios, as shown in Figure 3. From the results, it is confirmed that the proposed scheme can provide cell-specific bias values that form a tessellation highly consistent with the optimal one, while conventional schemes are not sufficiently consistent.

In Figures 4-6, some typical UA association examples of the joint UA scheme, the proposed scheme, and the common bias scheme are illustrated for the three scenarios, respectively. A comparison among the user association results can be summarized as follows: (i) compared with the joint UA scheme, a similar UA association can be achieved by using the proposed scheme without considering the user locations jointly, while the UA association obtained by using 


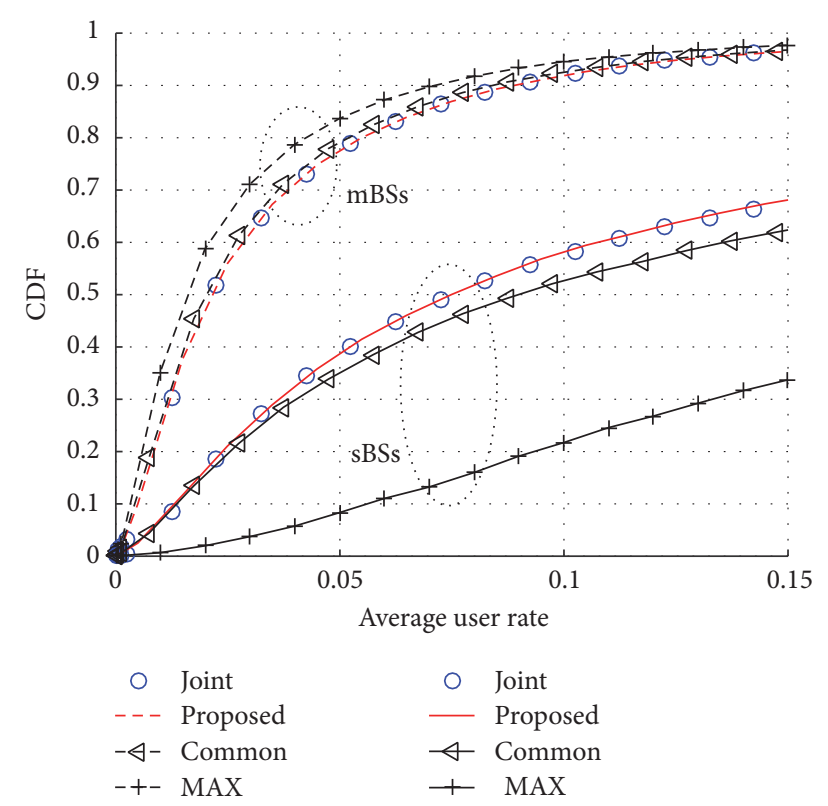

FIGURE 7: User rate distribution according to load-balancing capabilities in urban scenario.

the common bias scheme deviates significantly, especially for sBSs near an mBS as consistently seen in Figures 4-6; (ii) although it seems that lightly loaded sBSs not close to an mBS can expand aggressively even by using the common bias scheme in the rural scenario, as seen in Figure 4, such lightly loaded sBSs not close to an $\mathrm{mBS}$ begin to fail when expanding their ranges successfully in the urban scenario, as seen in Figure 5; and (iii) they are barely expanded in the dense urban scenario, as seen in Figure 6. Especially in the dense urban scenario, it is shown that the proposed scheme can provide a flexible intratier offloading (between sBSs in a hotspot) as well as an aggressive intertier offloading (from mBSs to sBSs) similar to those achieved in the joint UA scheme, while the common bias scheme suffers from almost no offloading between sBSs and limited intertier offloading.

In Figure 7, the load-balancing capability of the proposed scheme is evaluated and compared with the joint UA and common bias schemes for the urban scenario in terms of the cumulative distribution function (CDF) of the user average rate. From the results, it is confirmed that the improved consistency in the tessellation significantly enhances the user rate distribution. Note that the user rate distribution achieved by the load-balancing capability of the proposed scheme is significantly better than that obtained from the common bias scheme and is even indistinguishable from that of the optimal joint UA scheme considering the user locations jointly.

In Figure 8, the average performance gain of the proposed scheme over the common bias scheme in terms of the ratio of the bottom $5 \%, 10 \%$, and $15 \%$ average user rates is evaluated and compared for the three realistic HCN scenarios. This analysis shows a significant improvement in network-wide utilization, especially as the traffic demand and the corresponding deployment become denser.

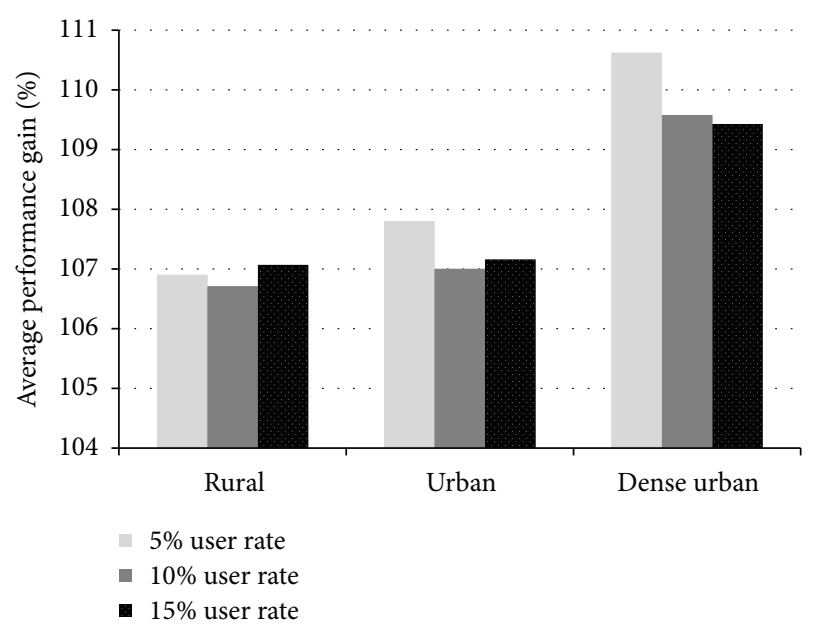

FigURE 8: Average performance gain of proposed scheme over common bias scheme in various $\mathrm{HCN}$ scenarios.

\section{Concluding Remark}

In this paper, a practical tessellation-based approach for optimizing cell-specific biases is proposed to improve the performance of existing LTE-A HCNs. In this approach, (i) based on long-term accumulated user measurement information, cell-specific bias values are optimized and updated globally by the EPC-MME to form a suboptimal tessellation in a long-term manner and are infrequently delivered to sBSs, and (ii) the ABS ratio and $\mathrm{ABS} / \mathrm{NS}$ resource scheduling of each mBS and its associated sBSs are determined locally and independently. The proposed scheme does not require major changes to existing protocol and can adopt legacy UEs. Thus, the scheme is well suited for implementation in existing LTE-A systems. From the simulation results in realistic scenarios, it is shown that the proposed scheme is very efficient in forming cell coverage that is as similar as possible to the optimal tessellation so that the performance in terms of the user rate distribution is almost identical to that achievable from an ideal one. Thus, it is beneficial to employ the proposed solution in existing LTE-A HCNs for better network performance during system updates at low cost.

\section{Conflicts of Interest}

The authors declare that there are no conflicts of interest regarding the publication of this paper.

\section{Acknowledgments}

This research was supported by Basic Science Research Program through the National Research Foundation of Korea (NRF) funded by the Ministry of Education, Science and Technology (NRF-2014R1A2A2A01007254 and NRF2015K2A3A1000189) in part and Institute for Information \& Communications Technology Promotion (IITP) grant funded by the Korea Government (MSIP) (2014-0-00552, 
Next Generation WLAN System with High Efficient Performance) in part.

\section{References}

[1] R. Q. Hu, Y. Qian, S. Kota, and G. Giambene, "Hetnets-a new paradigm for increasing cellular capacity and coverage," IEEE Wireless Communications, vol. 18, no. 3, pp. 8-9, 2011.

[2] J. G. Andrews, S. Singh, Q. Ye, X. Lin, and H. S. Dhillon, "An overview of load balancing in HetNets: old myths and open problems," IEEE Wireless Communications, vol. 21, no. 2, pp. 1825, 2014.

[3] S. Corroy, L. Falconetti, and R. Mathar, "Cell association in small heterogeneous networks: downlink sum rate and min rate maximization," in Proceedings of the IEEE Wireless Communications and Networking Conference, WCNC 2012, pp. 888-892, Shanghai, China, April 2012.

[4] H. Kim, G. De Veciana, X. Yang, and M. Venkatachalam, "Distributed-optimal user association and cell load balancing in wireless networks," IEEE/ACM Transactions on Networking, vol. 20, no. 1, pp. 177-190, 2012.

[5] Y. Wang and K. I. Pedersen, "Performance analysis of enhanced inter-cell interference coordination in LTE-advanced heterogeneous networks," in Proceedings of the IEEE 75th Vehicular Technology Conference, VTC Spring 2012, June 2012.

[6] K. I. Pedersen, Y. Wang, B. Soret, and F. Frederiksen, "eICIC functionality and performance for LTE HetNet co-channel deployments," in Proceedings of the 76th IEEE Vehicular Technology Conference (VTC Fall '12), pp. 1-5, IEEE, Quebec City, Canada, September 2012.

[7] M. Shirakabe, A. Morimoto, and N. Miki, "Performance evaluation of inter-cell interference coordination and cell range expansion in heterogeneous networks for LTE-Advanced downlink," in Proceedings of the 2011 8th International Symposium on Wireless Communication Systems, ISWCS 2011, pp. 844848, Aachen, Germany, November 2011.

[8] Q. Ye, M. Al-Shalashy, C. Caramanis, and J. G. Andrews, “On/off macrocells and load balancing in heterogeneous cellular networks," in Proceedings of the IEEE Global Communications Conference, GLOBECOM 2013, pp. 3814-3819, IEEE, Atlanta, Ga, USA, December 2013.

[9] W. Tang, R. Zhang, Y. Liu, and S. Feng, "Joint resource allocation for eICIC in heterogeneous networks," in Proceedings of the 2014 IEEE Global Communications Conference, GLOBECOM 2014, pp. 2011-2016, December 2014.

[10] S. Deb, P. Monogioudis, J. Miernik, and J. P. Seymour, "Algorithms for enhanced inter-cell interference coordination (eICIC) in LTE HetNets," IEEE/ACM Transactions on Networking, vol. 22, no. 1, pp. 137-150, 2014.

[11] Q. Zhang, T. Yang, Y. Zhang, and Z. Feng, "Fairness guaranteed novel eICIC technology for capacity enhancement in multi-tier heterogeneous cellular networks," EURASIP Journal on Wireless Communications and Networking, vol. 2015, no. 1, 2015.

[12] B. Soret and K. I. Pedersen, "Centralized and distributed solutions for fast muting adaptation in LTE-Advanced HetNets," IEEE Transactions on Vehicular Technology, vol. 64, no. 1, pp. 147-158, 2015.

[13] H. Zhang, Y. Li, and Y. Li, "Traffic-based adaptive resource management for eICIC and CRE in heterogeneous networks," in Proceedings of the 2013 IEEE 24th International Symposium on Personal, Indoor and Mobile Radio Communications, PIMRC Workshops 2013, pp. 117-121, London, UK, September 2013.
[14] A. Tall, Z. Altman, and E. Altman, "Self organizing strategies for enhanced ICIC (eICIC)," in Proceedings of the 2014 12th International Symposium on Modeling and Optimization in Mobile, Ad Hoc, and Wireless Networks, WiOpt 2014, pp. 318325, Hammamet, Tunisia, May 2014.

[15] 3GPP., "Evolved universal terrestrial radio access (e-UTRA) and evolved universal terrestrial radio access network (e-UTRAN); overall description; stage 2," TS 36.300 version 10.11.0 Release 10, September 2013.

[16] Small cell forum, "Small cell services in rural and remote environments," version: 157.07.01, pp. 1-8, March 2015, http://www .smallcellforum.org/.

[17] Small cell forum, "Small cell services in the urban environment," version: 090.07.01, pp. 1-7, February 2014, http://www.smallcellforum.org/.

[18] R. Kreher and K. Gaenger, LTE Signaling: Troubleshooting and Optimization, Wiley, 2010, ISBN: 978-0-470-97767-5.

[19] R. W. Heath Jr., T. Wu, Y. H. Kwon, and A. C. Soong, "Multiuser MIMO in distributed antenna systems with out-ofcell interference," IEEE Transactions on Signal Processing, vol. 59, no. 10, pp. 4885-4899, 2011.

[20] CVX Research Inc., http://cvxr.com/, 2012.

[21] F. Xie, H. Nakhost, and M. Müller, "Planning via random walkdriven local search," in Proceedings of the 22nd International Conference on Automated Planning and Scheduling, ICAPS 2012, pp. 315-322, June 2012.

[22] 3GPP, "3rd generation partnership project; technical specification group GSM/EDGE radio access network; mobile radio interface layer 3 specification; radio resource control (RRC) protocol," TS 44.018 version 8.10.0 Release 8, March 2010.

[23] Ultra dense network (UDN), "Ultra dense network (UDN)," White Paper, pp. 1-26, 2016, http://resources.alcatel-lucent.com/ asset/200295.

[24] 3GPP, "LTE; evolved universal terrestrial radio access (EUTRA); radio frequency (RF) system scenarios," TR 36.942 version 10.2.0 Release 10, 2011.

[25] Real Wireless, An Assessment of the Value of Small Cell Services to Operators, Virgin Media Version 3.1, 2012.

[26] J. G. Andrews, F. Baccelli, and R. K. Ganti, "A tractable approach to coverage and rate in cellular networks," IEEE Transactions on Communications, vol. 59, no. 11, pp. 3122-3134, 2011.

[27] V. Fernandez-Lopez, K. I. Pedersen, and B. Soret, "Effects of interference mitigation and scheduling on dense small cell networks," in Proceedings of the 80th IEEE Vehicular Technology Conference, VTC 2014-Fall, can, September 2014.

[28] S. N. Chiu, D. Stoyan, W. S. Kendall, and J. Mecke, Stochastic Geometry And Its Applications, Wiley Series in Probability and Statistics, John Wiley \& Sons, Ltd., Chichester, 3rd edition, 2013, ISBN: 978-0-470-66481-0.

[29] T. Nakamura, S. Nagata, A. Benjebbour et al., "Trends in small cell enhancements in LTE advanced," IEEE Communications Magazine, vol. 51, no. 2, pp. 98-105, 2013.

[30] H. Klessig, V. Suryaprakash, O. Blume, A. Fehske, and G. Fettweis, "A framework enabling spatial analysis of mobile traffic hot spots," IEEE Wireless Communications Letters, vol. 3, no. 5, pp. 537-540, 2014.

[31] 3GPP, "3rd generation partnership project; Technical specification group radio access network; Evolved universal terrestrial radio access (e-UTRA); Mobility enhancements in heterogeneous networks," TR 36.839 version 11.0.0 Release 11, September 2012. 


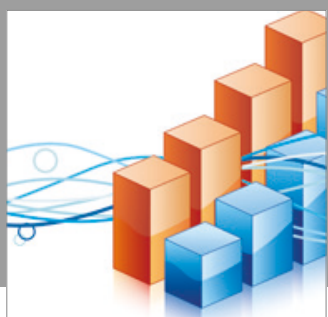

Advances in

Operations Research

vatersals

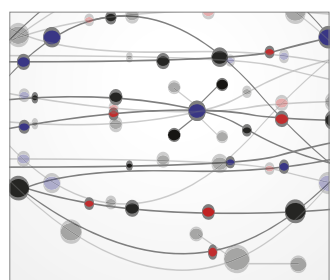

\section{The Scientific} World Journal
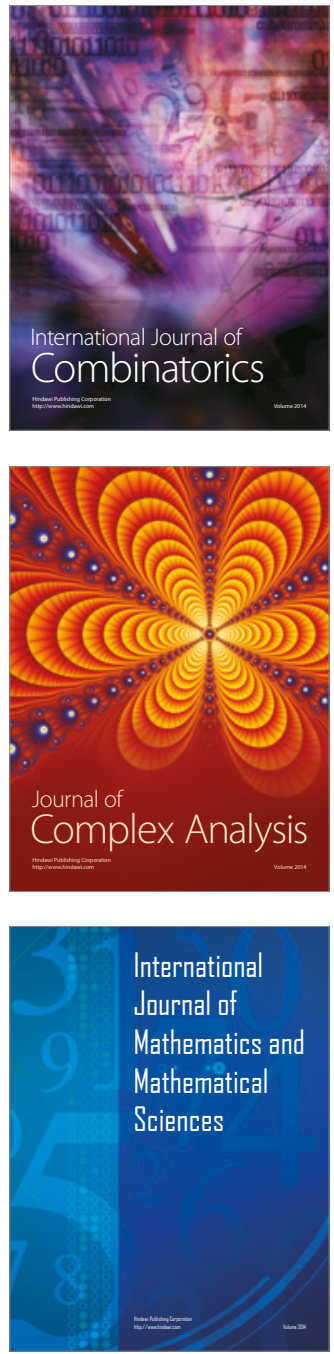
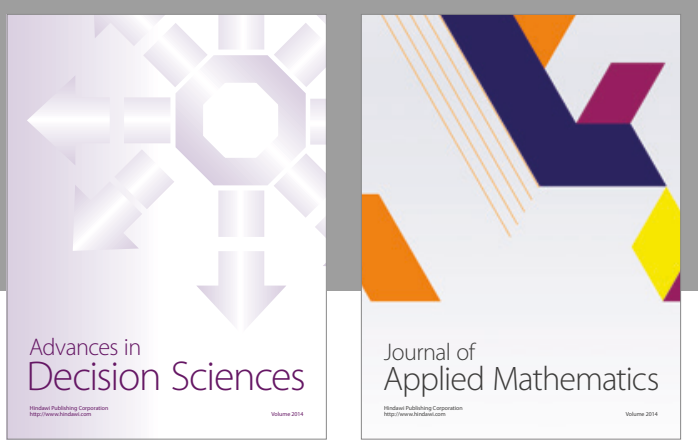

Algebra

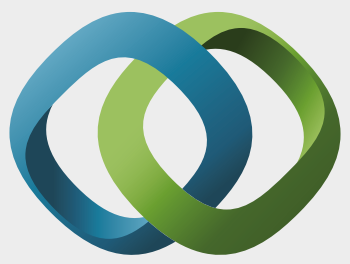

\section{Hindawi}

Submit your manuscripts at

https://www.hindawi.com
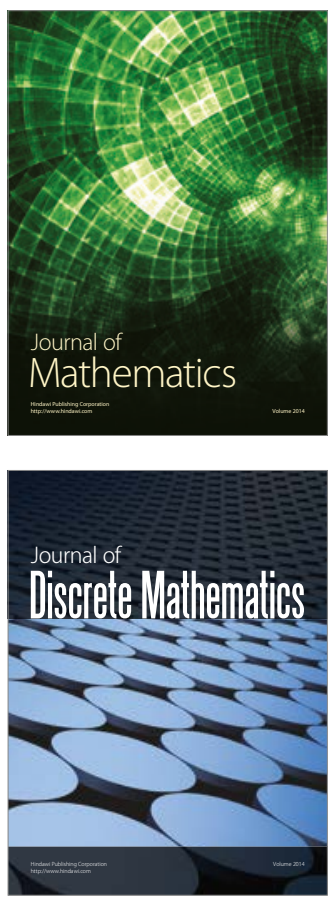

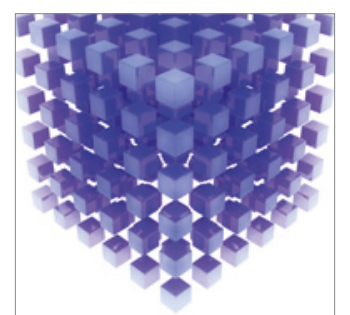

Mathematical Problems in Engineering
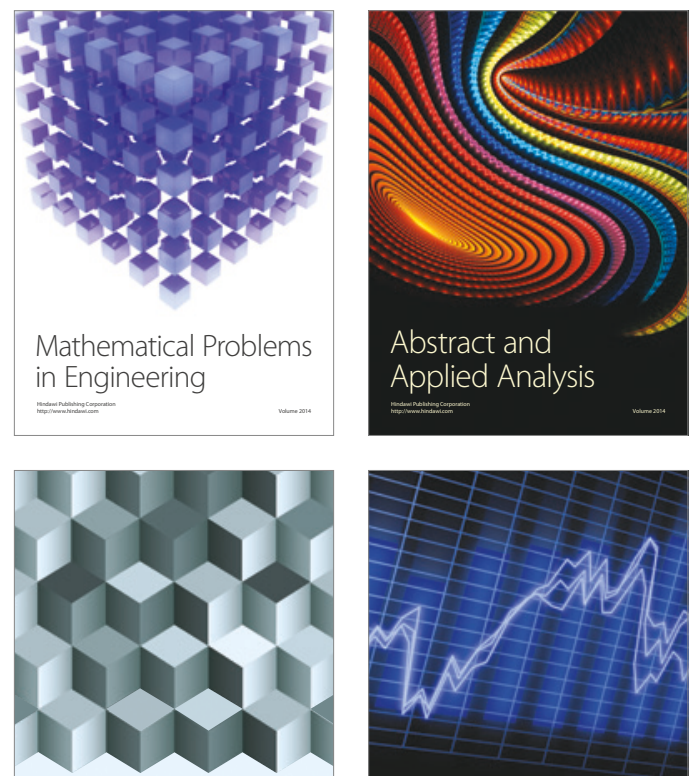

Journal of

Function Spaces

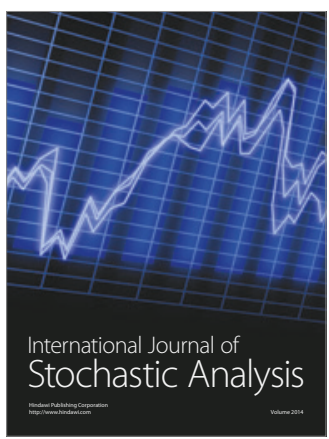

Probability and Statistics
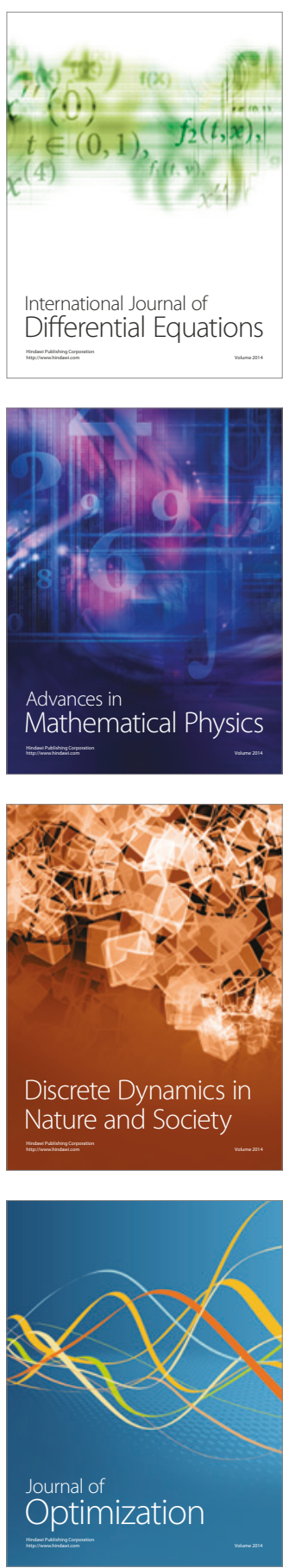